\title{
ON THE LOGARITHMIC PROXIMATE ORDER OF ANALYTIC FUNCTIONS OF SLOW GROWTH REPRESENTED BY LAPLACE-STIELTJES' TRANSFORMATIONS
}

\author{
ChHaya Singhal and Girja S. SRivastava
}

\begin{abstract}
In the present paper the characterizations of generalized type and generalized lower type of analytic functions represented by Laplace-Stieltjes transformation have been obtained. For this we introduce the concept of logarithmic proximate order.

Mathematics subject classification (2010): 30D15, 32A15.

Keywords and phrases: Laplace-Stieltjes transformations, order, proximate order, logarithmic type.
\end{abstract}

\section{REFERENCES}

[1] K. N. Awasthi AND K. K. DiXit, On The logarithmic order of analytic functions represented by Dirichlet series, Indian J. pure app. Math., 10 (2), (1979) 171-182.

[2] K. N. AWASTHI AND K. K. DiXIT, On the logarithmic proximate order of analytic functions represented by Dirichlet series, Indian J. pure app. Math., 11 (2), (1980), 1590-1599.

[3] K. N. Awasthi AND K. K. Dixit, On The logarithmic type of analytic functions represented by Dirichlet series, The Mathematics Student, 48 (4), (1980), 381-391.

[4] Xu Hongyan, Yi CAifeng And Ting Bin CAO, On proximate order and type function of LaplaceStieltjes' transformations convergent in the right half-plane, Math. Commun, 17 (2012), 355-369.

[5] YIN YING KONG AND SUN DaOchun, On type-function and the growth of Laplace-Stieltjes' transformations convergent in the right half plane, Advances in Math., 37, (2008), 197-205.

[6] Yin Ying Kong And Yan Yang, On the growth properties of Laplace-Stieltjes' transform, Complex Var. and Elliptic Equ., 59 (4), (2014), 553-563.

[7] Xi LuO AND YIN YING Kong, On the order and type of Laplace-Stieltjes transforms of slow growth, Acta Math. Sinica, 32A, (2012), 601-607.

[8] Chhaya Singhal and G. S. Srivastava, On the approximation of an analytic function represented by Laplace-Stieltjes' transformation, Anal. Theory Appl., 31 (4), (2015), 407-420.

[9] G. S. SRivastava AND ChHAYA Singhal, On the generalized order and generalized type of Laplace-Stieltjes transformation convergent in the right half-plane, Global Journal of Pure and Applied Mathematics 11 (1) (2015), 469-477.

[10] Lu Wanchun, On the proximate type of analytic functions represented by Laplace-Stieltjes' transformation, Jour. Math. Res. Appl., 35 (1), (2015), 97-102.

[11] Lu Wanchun AND Yi CAIFEnG, On logarithmic order and logarithmic proximate order of the analytic function defined by Laplace-Stieltjes' transformation, Acta Math. Sci., 31 (5), 2014, 12361244.

[12] JingJing YAn, On the orders and types of Laplace-Stieltjes transforms of slow growth in the right half plane, Acta Math. Sinica, 3, (2012), 1-12.

[13] JiARONG YU, Borel's line of entire functions represented by Laplace-Stieltjes transformations, Acta Math. Sinica, 13, (1963), 471-484. 